\title{
Intracranial Hypotension Secondary To A Cerebrospinal Fluid Leak
}

\author{
Ivan Urits, $\mathrm{MD}^{1}$, George Chesteen, $\mathrm{MD}^{2}$, Vwaire Orhurhu, $\mathrm{MD}^{1}$, Alan D. Kaye, $\mathrm{MD}, \mathrm{PhD}^{3}$, and \\ Omar Viswanath MD ${ }^{4-6}$
}

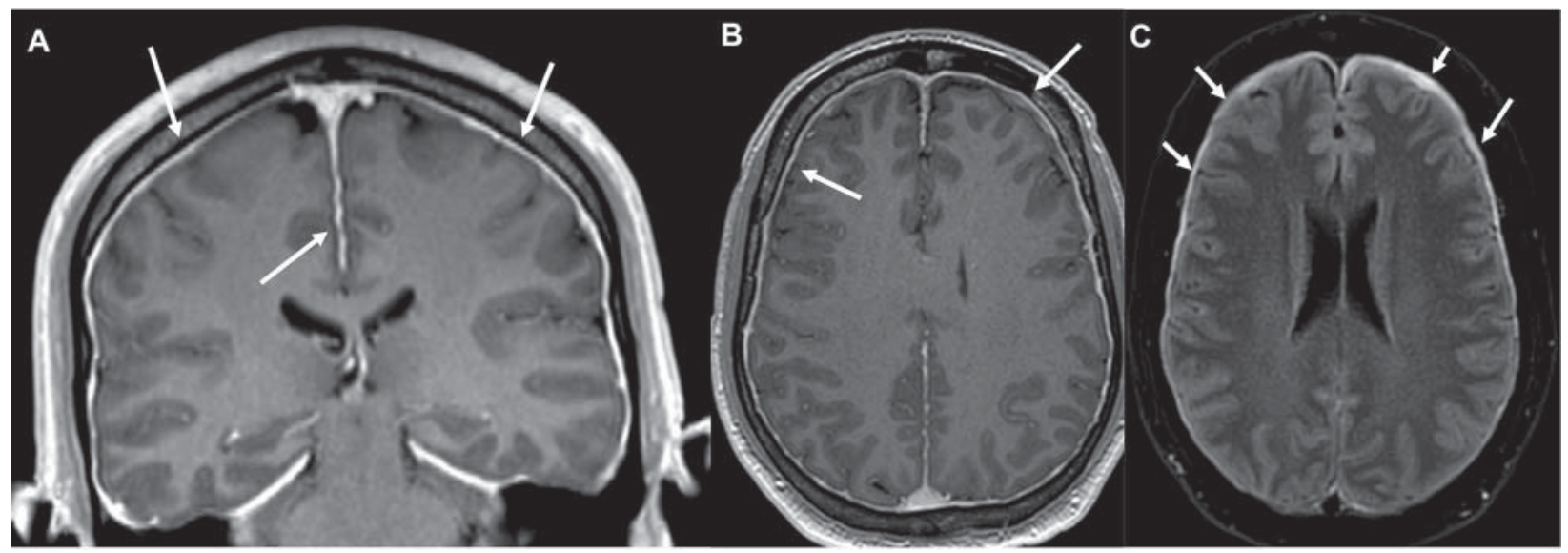

Cranial computed tomography (CT) imaging of a patient with spontaneous hypovolemic intracranial hypotension.

Fig 1: Coronal (a) and axial (b) T1 contrast enhanced CT images demonstrating diffuse circumferential dural enhancement secondary to venous engorgement. Axial FLAIR coronal CT image (c) demonstrating acute subdural fluid collections.

Spontaneous intracranial hypotension secondary to cerebrospinal fluid (CSF) leak is an important cause of severe headaches that are often refractory to conservative management. Many of the characteristic findings of intracranial hypotension can be explained by the Monro-Kellie hypothesis which defines the

From: ${ }^{1}$ Beth Israel Deaconess Medical Center, Department of Anesthesia, Critical Care, and Pain Medicine, Harvard Medical School, Boston, MA; ${ }^{2}$ Barrow Neurological Institute, Phoenix, AZ; ${ }^{3}$ Louisiana State University Health Sciences Center, Department of Anesthesiology, New Orleans, LA; ${ }^{4}$ Valley Anesthesiology and Pain Consultants, Phoenix, AZ; ${ }^{5}$ University of Arizona College of Medicine - Phoenix, Department of Anesthesiology, Phoenix, AZ; ; ${ }^{6}$ Creighton University School of Medicine, Department of Anesthesiology, Omaha, NE

Author for correspondence: Ivan Urits, MD

Address: Beth Israel Deaconess Medical Center

Department of Anesthesia, Critical Care, and Pain Medicine 330 Brookline Ave

Boston, MA, 02215

E-mail: iurits@bidmc.harvard.edu volume of brain matter, CSF, and intracranial blood as a necessary constant (1). In consideration of the intracranial space as a fixed volume, loss of CSF volume is met by compensating increases of subdural venous collections and intravascular venous blood. In the images presented, T1 weighted computed tomography (CT) demonstrates profound circumferential dural enhancement secondary to venous engorgement. Furthermore, FLAIR axial CT imaging captures subdural fluid collections secondary to transvenous hydrostatic pressure (Fig 1). While diagnosis continues to be difficult and largely based on clinical findings of postural headache symptoms, qualitative signs apparent on MRI such as dural enhancement, venous engorgement, and subdural fluid collections may offer improved accuracy in the diagnosis of intracranial hypotension (2). In patients nonresponsive to initial noninvasive treatments, symptomatic relief of headaches secondary to intracranial hypotension as a result of CSF leak may conventionally be achieved by epidural blood patch though efficacy and safety is remains to be investigated (3). 


\section{References}

1. Mokri B. The Monro-Kellie hypothesis: Applications in CSF volume depletion. Neurology 2001; 56:1746-1748.

2. Aslan K, Gunbey HP, Tomak L, Ozmen Z, Incesu L. Magnetic resonance imaging of intracranial hypotension. J Comput Assist
Tomogr 2017; $42: 1$.

3. Lin J, Zhang S, He F, Liu M, Ma X. The status of diagnosis and treatment to intracranial hypotension, including $\mathrm{SIH}$. J Headache Pain 2017;18:4. 